\title{
Image Processing of Corona Virus Using Interferometry
}

\author{
Abdallah Mohamed Hamed \\ Physics Department, Faculty of Science, Ain Shams University, Cairo, Egypt \\ Email: amhamed73@hotmail.com
}

Received 4 February 2016; accepted 27 May 2016; published 30 May 2016

Copyright (C) 2016 by author and Scientific Research Publishing Inc.

This work is licensed under the Creative Commons Attribution International License (CC BY). http://creativecommons.org/licenses/by/4.0/

c) (i) Open Access

\begin{abstract}
A new method of image processing of corona virus based on two and multiple beam interference is suggested. The method is based on measuring the fringe shift with respect to the background interference pattern. The interested application of the corona virus image in confocal microscopy is getting depth information since it has the property of optical sectioning. An accurate measurement of the fringe shift is obtained using multiple beam interference since contrast is higher than that for two beam interference. The refractive index of the corona virus image is deduced from the fringe shift. A MATLAB code is used for the processing of all images.
\end{abstract}

\section{Keywords}

Interference, Image Processing, Refractive Index Distribution of Corona Virus

\section{Introduction}

Corona viruses are an important family of human and veterinary pathogens that can cause enteric and respiratory infections. Corona virus infection can lead to respiratory failure, gastroenteritis, nephritis, and hepatitis.

A novel methodology of single particle image analysis is applied to select virus features in order to obtain detailed model of oligomer state and spatial relationships among viral structural proteins [1].

The addition of electronics, computers, and software to interferometry has enabled enormous improvements to optical metrology. Phase-shifting interferometry is used for getting data into a computer so the data can be analyzed [2]-[9]. Image processing of uniform objects and modified apertures was outlined [10]-[14].

In this paper, the refractive index distribution of the corona virus images is computed from the phase shift method. The corona virus fringe shift with respect to the background interference is computed to get useful information about the phase shift of the image leading map the height depth and the refractive index distribution. The results and discussions are given followed by a conclusion. The former work concerning the digital fringe shift is limited by $\lambda / 2$ the inter-fringe spacing. Hence, the computation of refractive index is dependent on the 
fringe spacing either using two or multiple beam interference.

\section{Analysis}

The complex amplitude of the corona virus as an object can be represented as follows:

$$
A_{o b j}(x, y ; z)=a \exp [i \Phi(x, y ; z)]
$$

where $a$-is the amplitude of the image, and $\Phi(x, y ; z)$ is its phase for object depth $z$. Equation (1) can be written in a discrete matrix form as follows:

$$
A_{o b j}(x, y ; z)=\sum_{n=1}^{N} \sum_{m=1}^{M} a \exp [i \Phi(n \Delta x, m \Delta y ; z)]
$$

where a square matrix of dimensions $N \times N=512 \times 512$ pixels $(N=M)$ is assumed.

This work focuses on the main technique for phase evaluation of the corona virus image using the phase shifting method.

Coherent addition of a reference laser beam $A_{r}=\operatorname{Rexp}[i \Psi(x, y)]$ to the above object beam is considered to fabricate the modeled interference pattern. The laser beam is spatially filtered using a pinhole located in the focal plane of a converging lens in order to get uniform illumination. In this case, a plane wave is obtained. The pinhole pass only the central peak from the whole diffraction pattern and suppress all the diffraction legs and then the converging lens which is placed a distance (f) from the pinhole passes parallel rays of uniform intensity which is considered as a plane wave.

Then the intensity of the two beam interference obtained in the detector plane can be expressed as the modulus square as follows:

$$
I(x, y ; z)=I_{0}\{1+M \cos [\Phi(x, y ; z)-\Psi(x, y)]\}
$$

where $I(x, y ; z)$ is the intensity of the modulated interference field at the point $I(x, y)$ for object depth $z$, $I_{0}=|R|^{2}+|a|^{2}$, is the function that characterizes the mean intensity of the interference pattern and $M=(2 a R) /\left[|R|^{2}+|a|^{2}\right]$, is the function that determines the modulation of the interference signal. In this case, the obtained trigonometric function has straight line fringes modulated by the object phase information. The modulated intensity is rewritten in matrix form as follows:

$$
I(x, y ; z)=I_{0} \sum_{n=1}^{N} \sum_{m=1}^{M}\{1+M \cos [\Phi(n \Delta x, m \Delta y ; z)-\Psi(n \Delta x, m \Delta y)]\}
$$

Certainly, the d. c term in equation (3), $I_{0}=|a|^{2}+R^{2}$.

$R$ is the amplitude of the coherent laser beam and that $\Psi$ appeared in equation (3) its phase. The equation (4) is used in the fabrication of the phase-shifted images outlined in equation (5).

Since the distance between any two fringes $=\lambda / 2$. Consequently, according to the phase shift technique [7] [9], the phase information of the image is governed by the following equation:

$$
\Psi(n \Delta x, m \Delta y)=\Phi(n \Delta x, m \Delta y ; z)-\tan ^{-1}\left[\frac{I_{3}(x, y)-I_{2}(x, y)}{I_{1}(x, y)-I_{2}(x, y)}\right]
$$

where the range of the interference phase $\Phi(n \Delta x, m \Delta y)$ extends from 0 up to $2 \pi$ for a height $z, I_{1}$ is the intensity given in equation (4) at a phase $\Psi=\pi / 2, I_{2}$ has $\Psi=\pi$, and $I_{3}$ has phase $\Psi=3 \pi / 2$. Then, three equations are solved to get equation (5).

Once the phase is determined across the interference field, the corresponding height distribution $h(x, y)$ on the surface of corona virus can be determined [2] as follows:

$$
h(x, y ; z)=\frac{\lambda}{4 \pi} \Phi(n \Delta x, m \Delta y ; z)
$$

We have assumed the surface measured at normal incidence. Almost all interferometers used to measure surface height variations use phase-shifting techniques.

The refractive index of the corona virus $\mu$ is computed as follows: 
Since the phase of the wave cumulates traveling a distance $L$ in a medium is

$$
\Phi(x, y)=\int_{L} k \mathrm{~d} l=\int \frac{\mu(x, y, z) \omega}{c} \mathrm{~d} l
$$

Then, the same wave that propagates over two equivalent paths $L$ in corona virus medium and in vacuum gives the phase difference as follows (Figure 1):

$$
\Delta \Phi(x, y ; z)=\int\left(k-k_{0}\right) \mathrm{d} l=\int[\mu(x, y, z)-1] \frac{\omega}{C} \mathrm{~d} l
$$

where $k=\omega / c=2 \pi / \lambda$ is the propagation wave number in a medium of refractive index $\mu$ while $k_{0}$ is the propagation constant in vacuum.

By differentiation w.r.t. the path $l=z$, the refractive index distribution of the corona virus image is computed as follows:

$$
\varpi(x, y)=1+\frac{c}{\omega} \frac{\mathrm{d}}{\mathrm{d} z}[\Delta \Phi(x, y ; z)]
$$

Since the angular frequency is related to the wavelength as $\omega=2 \pi c / \lambda$ and $\Delta \Phi(x, y)=\left(\frac{2 \pi}{\lambda}\right)($ O.P.D. $)$ then the above equation becomes:

$$
\mu(x, y)=1+\frac{\mathrm{d}}{\mathrm{d} z}[O . P . D .]
$$

The optical path difference represents the height variation of the image, namely $h(x, y, z)$ then equation (10) becomes:

$$
\mu(x, y)=1+\frac{\mathrm{d}}{\mathrm{d} z}[h(x, y, z)]
$$

The differentiation of the height distribution $h(x, y, z)$ with respect to $z$ give the differential fringe shift and the amplitude of the planar image $a(x, y)$. Consequently, we finally get equation (12).

$$
\mu(x, y)_{\text {const. } x}=1+a(x, y) \delta z / z
$$

The fringe shift is $\delta z$ with respect to inter-fringe spacing $\Delta z$ at constant $x$, the fringes are assumed located in the $x-y$ plane and $z$ is the axis normal to the fringe system which represents the height depth and $a(x, y)$ represents the amplitude of the image. In equation (12), $h(x, y, z)=a(x, y) \cdot \delta z$.

\section{Computation of the Contrast}

Consider the visibility expression to represent the fringe contrast as follows:

$C=\frac{I_{\max }-I_{\min }}{I_{\max }+I_{\min }}$. Substitute in equation (3), we get this result for the contrast:

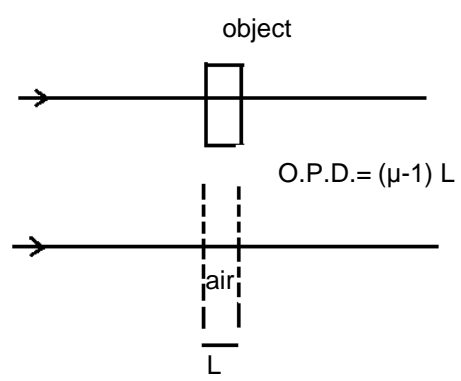

Figure 1. The propagation of light in a medium of refractive index $\mu$ compared with air. 


$$
C=M=2 R /\left(1+R^{2}\right)
$$

While the contrast given in case of multiple beam interference is extracted from the transmitted intensity distribution [15]:

$$
I_{t}=\frac{I_{0}}{1+F \sin ^{2}[\Phi(x, y)-\Psi(x, y)] / 2}
$$

The parameter $F=4 R /(1-R)^{2}$ and the corresponding contrast is given by the formula (11). The maximum and minimum intensities are obtained from equation (13) as:

$I_{\max }=I_{0}$ for $[\Phi(x, y)-\Psi(x, y)]=2 m \pi, m$ is integer.

$I_{\min }=I_{0} /(1+F)$ for $[\Phi(x, y)-\Psi(x, y)]=m \pi$

It is known that the refractive index has a direct relation with the polychromatic spectral distribution of illuminating light according to the Cauchy formula as follows:

$$
\mu_{\lambda}=a+b / \lambda^{2}
$$

where $a$, and $b$ are constants. Then the fringe shift and the refractive index are affected by the change of the wavelength.

\section{Results and Discussion}

The Corona virus image used in the processing is shown as in the Figure 2. It has dimensions of $512 \times 512$ pixels. The fringe shift occurred within the corona virus w.r.t. the background shift is plotted as in the Figure 3 at frequency $f=1 / 32$. This interferometer plotis obtained from equation (3) written in discrete form where $\mathrm{M}=N=$ 512 pixels. The yellow discontinuous horizontal lines are taken at 10, 80, 140, 200, 260, 320, 380, 440 pixels. Only 7 fringes are shown in this image. The shift of the corona virus cells are computed as compared with the inter-fringe spacing. The Mat Lab code is used for the computation of the modulation term $\cos (y-(1 / 32) A(i, j))$. While the background two beam interference is only cos (y). It is represented by straight line fringes modulated by the object matrix A (M, N). From the Figure 3, eight plots are shown in the Figure 4 at 10, 80,140, and 200 pixels in the $1^{\text {st }}$ column, and at 260, 320, 380, and 440 pixels in the $2^{\text {nd }}$ column. The upper left plot at 10 pixels show uniform straight line fringes which is compared with the shifted fringes corresponding to the image geometry shown at the mentioned lines. Hence, we can get phase information about the image as plotted in the Figure 5 using equation (8) and height information from equation (9).

Four different modulated fringes shifts are shown in the Figure 6. Interferometry images of the corona virus

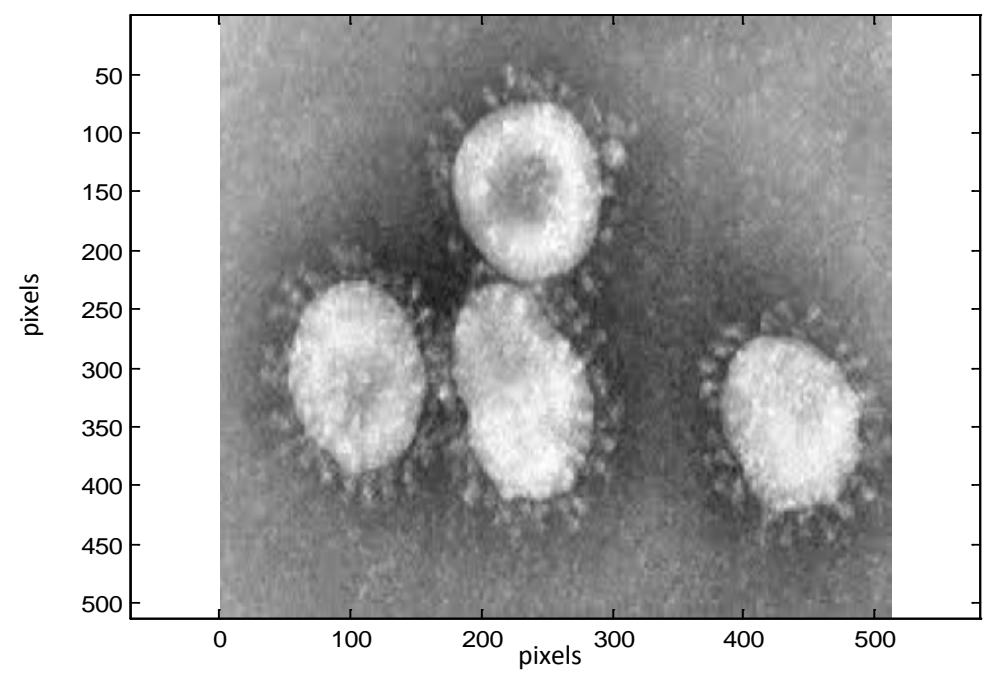

Figure 2. Corona virus image used in the processing. It has dimensions of $512 \times 512$ pixels. 


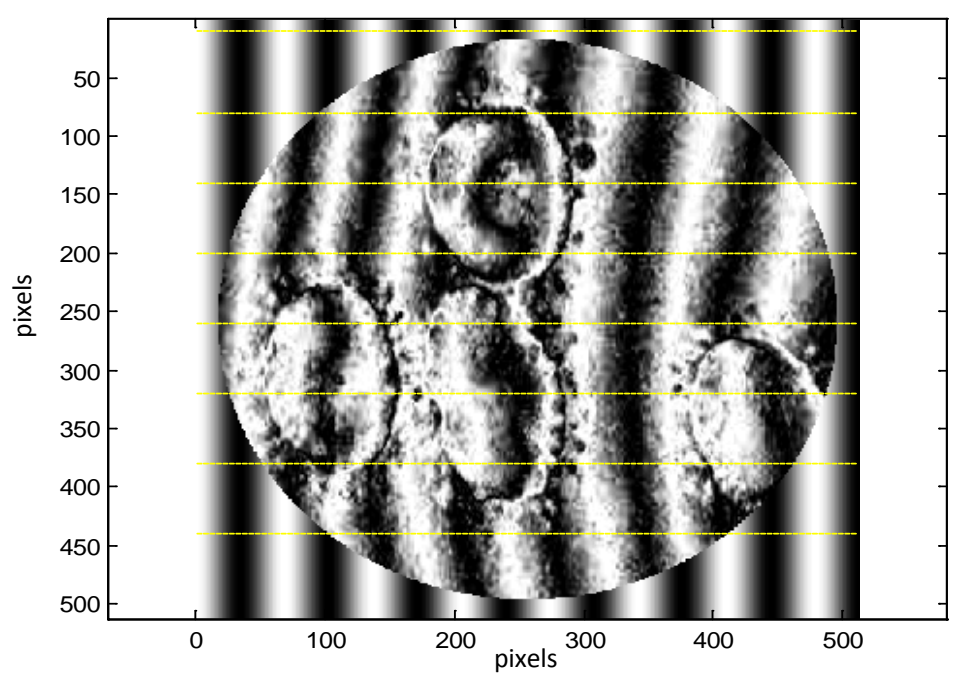

Figure 3. Two beam interference of corona virus image of dimensions $512 \times 512$ pixels. The yellow discontinuous lines are taken at $10,80,140$, 200, 260, 320, 380, 440 pixels. 7 fringes are shown in this image. The shift of the corona virus cells are computed compared with the inter-fringe spacing.
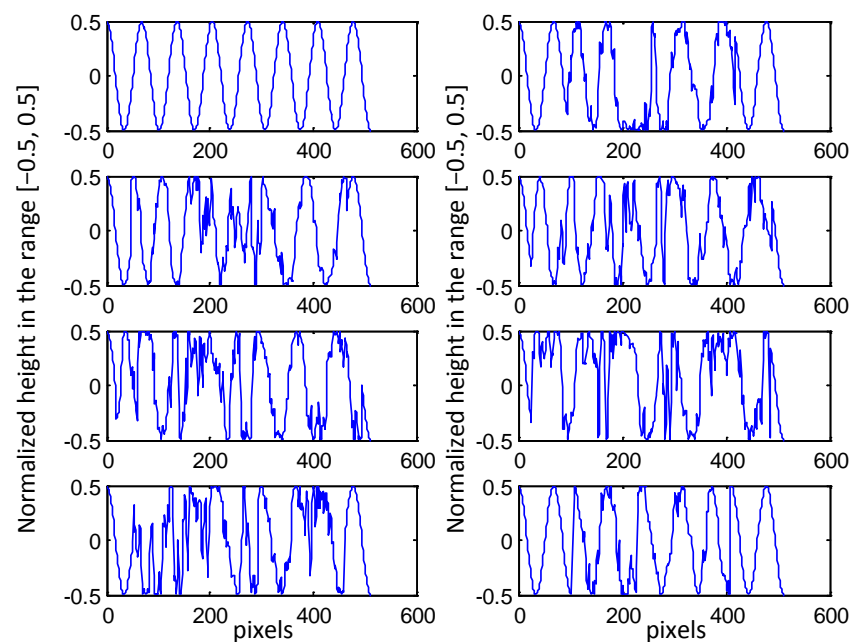

Figure 4. Eight plots from the Figure 3 are shown at 10, 80,140, and 200 pixels in the 1 st column in the left, and at $260,320,380$, and 440 pixels in the $2^{\text {nd }}$ column.

using the cosine function to represent the phase at four different spatial frequencies at 1/32, 1/64, 1/96, and 1/128. The original image is multiplied by a factor of $\alpha=1 / 32$ in the interference modulated terms as follows: for a) $\cos (y-(1 / 32) A(i, j)), b) \cos (2 y-(1 / 32) A(i, j))$, c) $\cos (3 y-(1 / 32) A(i, j))$, and d) $\cos (4 y-(1 / 32) A(i, j))$.

The multiple beam interferometry images of the corona virus using the Airy function to represent the phase at four different background frequencies is plotted as in the Figure 7. The original image is multiplied by a factor of $\alpha=1 / 32$ as in the Figure 6 .

Finally, comparing the fringe shift in the corona virus image at different frequencies $f=1 / 32,1 / 64,1 / 96$, and $1 / 128$, it is shown that the fringe shift is not resolved at frequency greater than $f=1 / 128$.

The profile of the corona virus image taken at constant $x=150$ pixels extracted from the image shown in the Figure 3 is plotted as in the Figure 8(a) and the profile taken at $x=330$ pixels is plotted in the Figure 8(b). In the left of both plots, seven fringes are shown. The profiles are affected by the fringe shift resulted from the phase change of the image under consideration. 


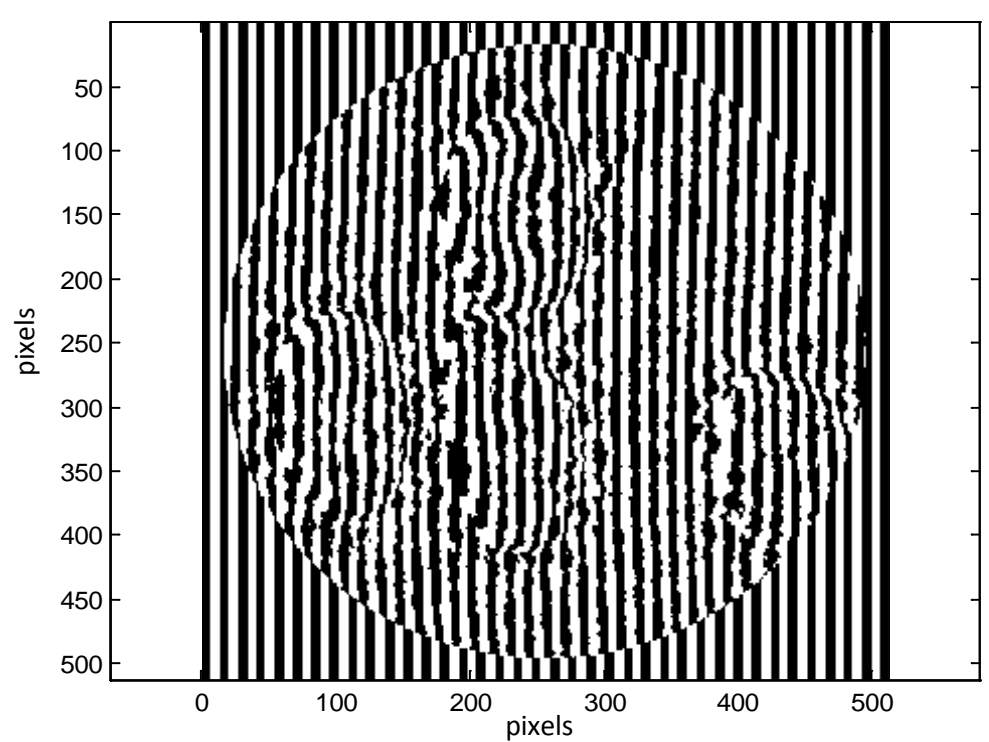

Figure 5. The Phase map of the corona virus of background spatial frequency $\mathrm{f}=5$; where $\alpha=1 / 32$.
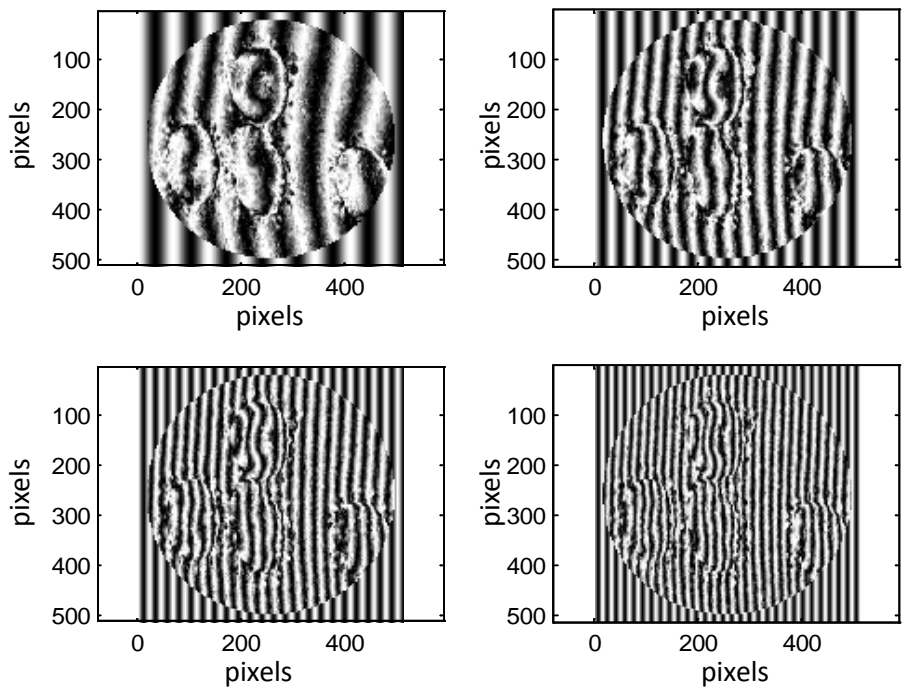

Figure 6. The interferometry images of the corona virus using the cosine function to represent the phase at four different spatial frequencies at $1 / 32$, $1 / 64,1 / 96$, and $1 / 128$. The original image is multiplied by a factor of $\alpha=$ $1 / 32$.

Effect of mirror reflection coefficient upon the multiple beam interference images is shown as in the Figure 9 . Refer to the derived equation from the Airy distribution (14); the contrast of the image is sharper for higher reflectivity, equation (13). The images shown in the Figure 9 are in good agreement with the theoretical results since higher contrast is attained at $R=80 \%$ as compared with that obtained at $\mathrm{R}=40 \%$.

The values of refractive index extracted from the Figure 10 are given in the Table 1 and plotted for horizontal line at 330 pixels as in the Figure 11. The modulated interference image of corona virus as in the Figure 12 where 32 fringes are plotted is used in the segmentation.

The upper cell segment selected from the image in the Figure 12 is shown as in the Figure 13(a) and the corresponding modulated fringe system of 11 fringes shown in the Figure 13(b) is investigated. The values of refractive index extracted from the Figure 13(b) for the cell are computed from equation (12) given in the Tables 2-4 and plotted for horizontal lines at 60, 80, and 100 pixels as in the Figure 14. 


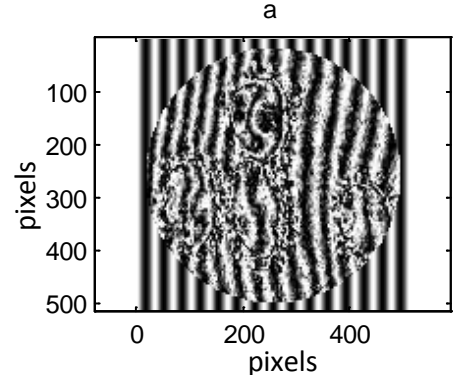

c

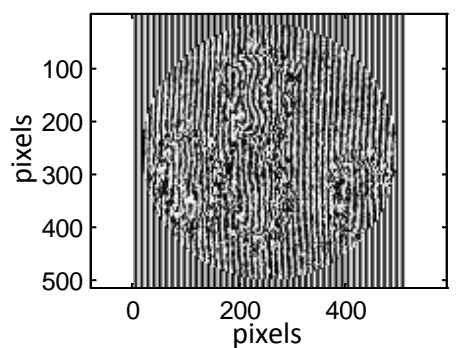

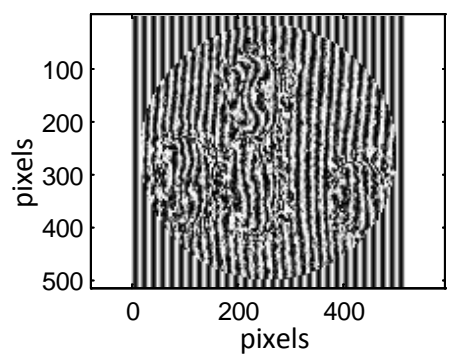

d

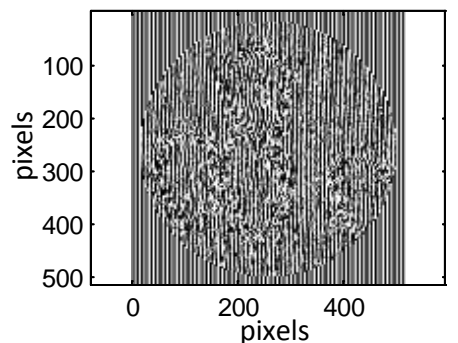

Figure 7. The multiple beam interferometry images of the corona virus using the Airy function to represent the phase at four different background frequencies as in the Figure 6 . The original image is multiplied by a factor of $\alpha=1 / 32$.
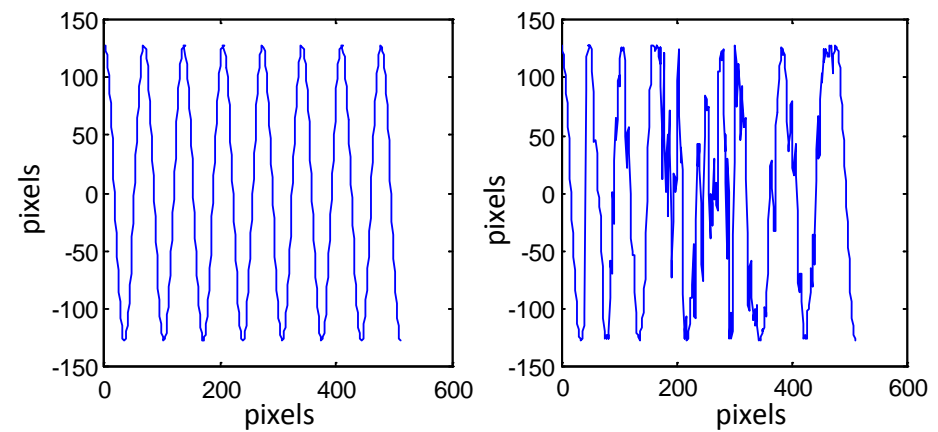

(a)
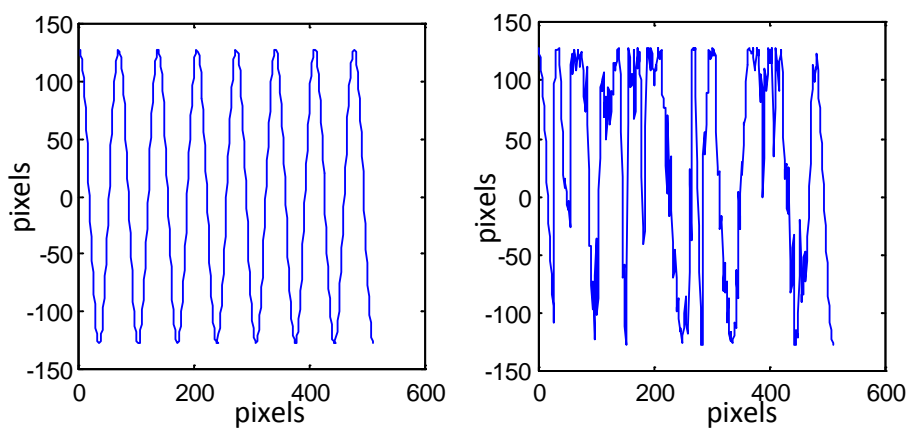

(b)

Figure 8. (a): The profile of the corona virus image taken at constant $x$ $=150$ pixels extracted from the image shown in the Figure 3. Seven fringes are shown; (b) the profile of the corona virus image taken at constant $x=330$ pixels extracted from the image shown in the Figure 3 . Seven fringes are given. 

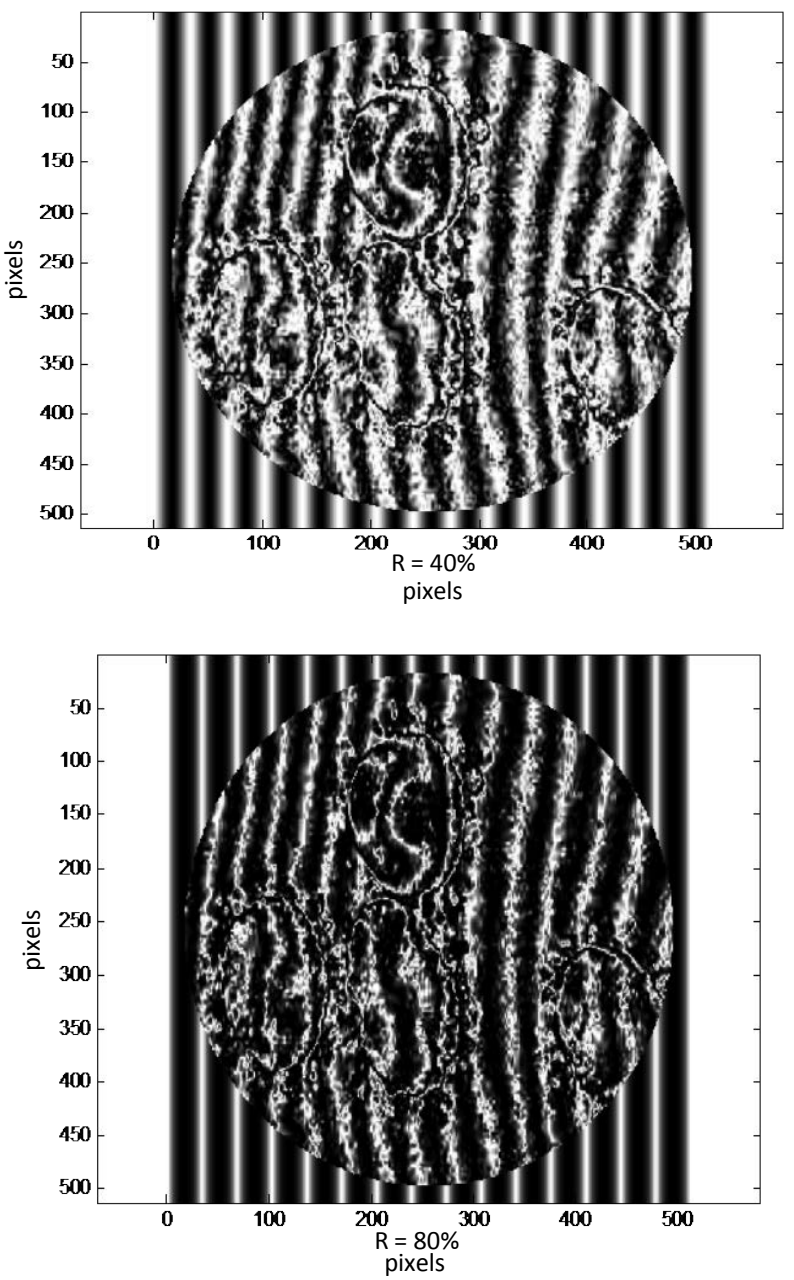

Figure 9. Effect of mirror reflection coefficient upon the multiple beam interference images is shown.

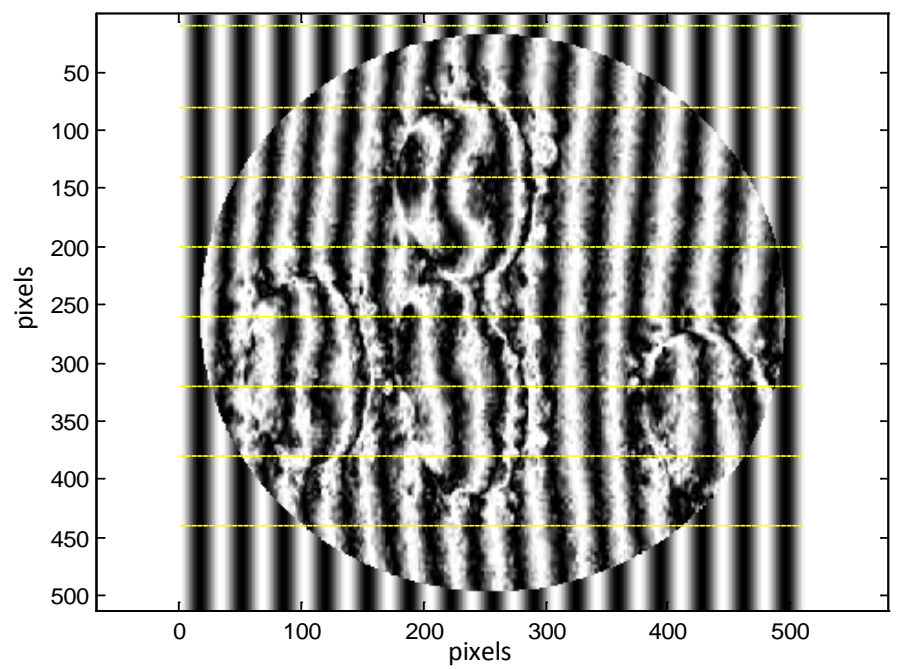

Figure 10. The corona virus image modulated by fourteen fringes used in the computation of the refractive index at 150 and 330 pixels as in the Table 1. 
Table 1. The refractive index values as a function of the $Z$ coordinate at certain horizontal line at 330 pixels.

\begin{tabular}{cccc}
\hline$Z$ & $Z_{\text {image }}$ & $\delta Z=Z-Z_{\text {image }}$ & $\mu(Z)=1+\delta Z / \Delta Z$ \\
\hline 34 & 2 & 32 & 1.94 \\
68 & 49 & 19 & 1.56 \\
102 & 99 & 3 & 1.09 \\
136 & 131 & 5 & 1.15 \\
170 & 158 & 12 & 1.35 \\
204 & 180 & 24 & 1.71 \\
272 & 272 & 0 & 1.00 \\
306 & 291 & 15 & 1.44 \\
340 & 319 & 21 & 1.62 \\
374 & 354 & 20 & 1.59 \\
408 & 384 & 24 & 1.71 \\
443 & 441 & 2 & 1.06 \\
479 & 476 & 3 & 1.09 \\
\hline
\end{tabular}

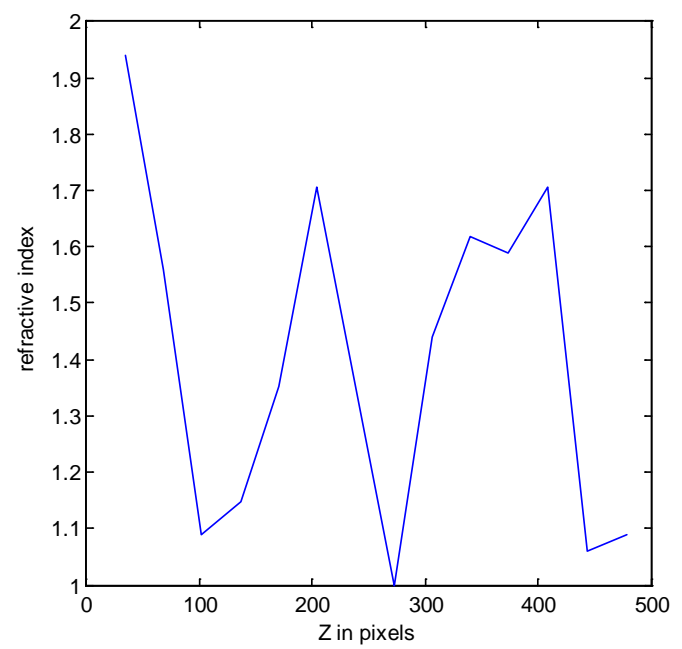

Figure 11. plot of the refractive index versus the horizontal distance at constant line $\mathrm{x}=330$ pixels. Three cells from the corona virus are scanned in the image. Inter-fringe spacing $\Delta Z=34$ pixels.

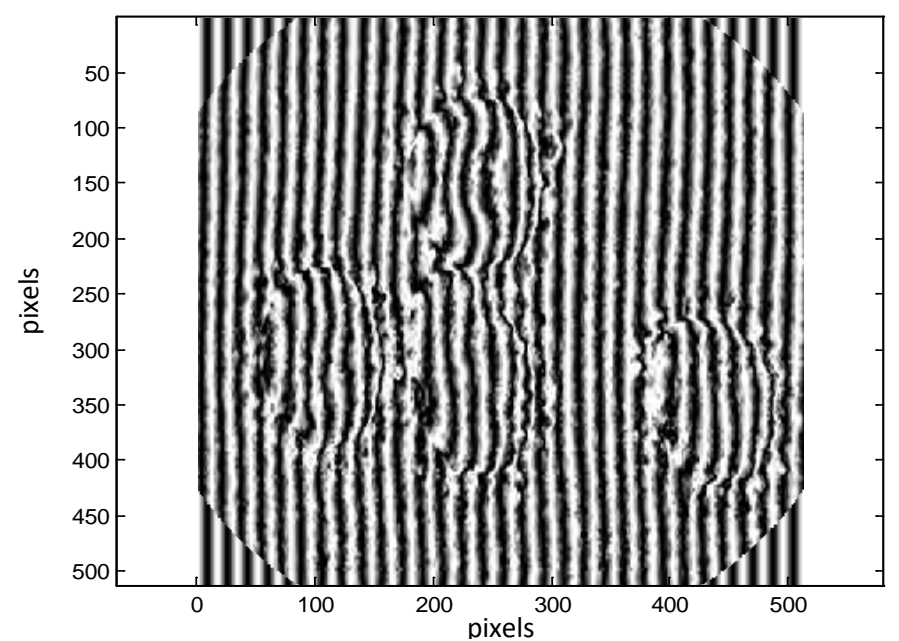

Figure 12. The modulated interference image of corona virus where 32 fringes are shown. 

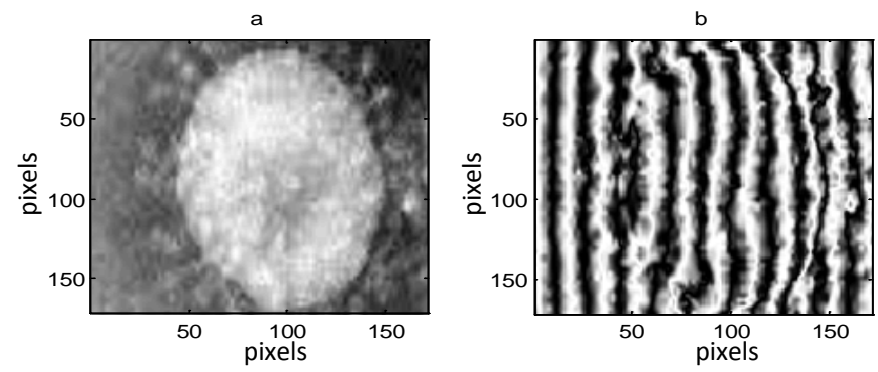

Figure 13. In the left (a), a segment from the image shown in the Figure 12 is taken at $i=220$ : 390 pixels and $j=10: 180$ pixels. The modulated two beam interference of the cell (segment) shown in (b) is investigated. Both segments in a, b have dimensions of $170 \times 170$ pixels.

Table 2. The refractive index values as a function of the $Z$ coordinate at certain horizontal line at 60 pixels.

\begin{tabular}{cccc}
\hline$Z$ & $Z_{\text {image }}$ & $\delta Z=Z_{\text {image }}-Z$ & $\mu(Z)=1+\delta Z / \Delta Z$ \\
\hline 51 & 63 & 12 & 1.80 \\
69 & 80 & 11 & 1.73 \\
84 & 96 & 12 & 1.80 \\
100 & 111 & 11 & 1.73 \\
118 & 126 & 8 & 1.53 \\
132 & 141 & 9 & 1.60 \\
149 & 152 & 3 & 1.20 \\
166 & 167 & 1 & 1.07 \\
\hline
\end{tabular}

Table 3. The refractive index values as a function of the $\mathrm{Z}$ coordinate at certain horizontal line at 80 pixels.

\begin{tabular}{cccc}
\hline$Z$ & $Z_{\text {image }}$ & $\delta Z=Z_{\text {image }}-Z$ & $\mu(Z)=1+\delta Z / \Delta Z$ \\
\hline 51 & 62 & 11 & 1.73 \\
69 & 76 & 7 & 1.47 \\
84 & 92 & 8 & 1.53 \\
100 & 108 & 8 & 1.53 \\
118 & 126 & 8 & 1.53 \\
132 & 141 & 9 & 1.60 \\
149 & 153 & 4 & 1.27 \\
166 & 170 & 4 & 1.27 \\
\hline
\end{tabular}

Table 4. The refractive index values as a function of the $Z$ coordinate at certain horizontal line at 100 pixels.

\begin{tabular}{cccc}
\hline$Z$ & $Z_{\text {image }}$ & $\delta Z=Z_{\text {image }}-Z$ & $\mu(Z)=1+\delta Z / \Delta Z$ \\
\hline 51 & 60 & 9 & 1.60 \\
69 & 75 & 6 & 1.40 \\
84 & 91 & 7 & 1.47 \\
100 & 107 & 7 & 1.47 \\
118 & 128 & 10 & 1.67 \\
132 & 144 & 12 & 1.80 \\
149 & 154 & 5 & 1.33 \\
166 & 171 & 5 & 1.33 \\
\hline
\end{tabular}



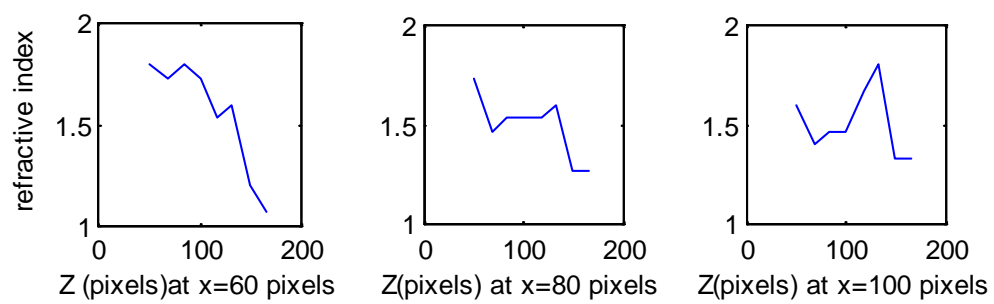

Figure 14. Three plots of the refractive index variation with $Z$ at constant horizontal lines at $x=60,80,100$ pixels computed from the Figure 13(b) which has only one cell.

The map of the refractive index distribution computed from equation (10) is the final object of this work.

\section{Conclusions}

The phase shift of corona virus images deduced from the interferometer images. The interferometer images using multiple beam interference gave better contrast than the corresponding images with the two-beam interference as expected. The effect of mirror reflection coefficient upon the multiple beam interference images discussed.

Useful information obtained from studying this virus using interferometry is extracted from the fringe shift of the modulated interference pattern namely the refractive index distribution of the whole image. Consequently, detailed and precise information about the virus may be extracted from the refractive index distribution. In addition, since the refractive index has a direct relation with the polychromatic spectral distribution of illuminating light according to the Cauchy formula it allows observe the diameter of the virus cell accurately as it changes with the wavelength of light.

\section{References}

[1] Neuman, B.W., Adair, B.D. and Virology, J. (2006) Super Molecular Architecture of Severe Acute Respiratory Syndrome Corona Virus Revealed by Electron Cryo-Microscopy. Journal of Virology, 80, 7918-7928. http://dx.doi.org/10.1128/JVI.00645-06

[2] Wyant, J.C. (2013) Computerized Interferometric Surface Measurements. Applied Optics, 52, 1-8. http://dx.doi.org/10.1364/AO.52.000001

[3] Crane, R. (1969) Interference Phase Measurement. Applied Optics, 8, 538-542.

[4] Wyant, J.C. (1973) Double Frequency Grating Lateral Shear Interferometer. Applied Optics, 12, 2057-2060. http://dx.doi.org/10.1364/AO.12.002057

[5] Bruning, J.H., Herriott, D.R., Gallagher, J.E., et al. (1974) Digital Wave-Front Measuring Interferometer for Testing Optical Surfaces and Lenses. Applied Optics, 13, 2693-2703. http://dx.doi.org/10.1364/AO.13.002693

[6] Wyant, J.C. (1975) Use of an Ac Heterodyne Lateral Shear Interferometer with Real-Time Wave Front Correction Systems. Applied Optics, 14, 2622-2626. http://dx.doi.org/10.1364/AO.14.002622

[7] Liu, J.P. and Poon, T.C. (2009) Two-Step-Only Quadrature Phase Shifting Digital Holography. Optics Letters, 34, 250-252. http://dx.doi.org/10.1364/OL.34.000250

[8] Yatagai, T. and Nakadate, S. (1982) Automatic Fringe Analysis Using Digital Image Processing Technique. Optical Engineering, 21, 432-435.

[9] Hamed, A.M. and Saudy, M.A. (2015) Image Processing of Argon Glow Discharge Plasma Using Interferometry. Journal of Plasma Physics, 81, 1-14. http://dx.doi.org/10.1017/S0022377815000550

[10] Hamed, A.M. (2009) Numerical Speckle Images Formed by Diffusers Using Modulated Conical and Linear Apertures. Journal of Modern Optics, 56, 1174-1181. http://dx.doi.org/10.1080/09500340902985379

[11] Hamed, A.M. (2009) Formation of Speckle Images Formed for Diffusers Illuminated by Modulated Apertures (Circular Obstruction). Journal of Modern Optics, 56, 1633-1642. http://dx.doi.org/10.1080/09500340903277792

[12] Hamed, A.M. (2011) Discrimination between Speckle Images Using Diffusers Modulated by Some Deformed Apertures: Simulation. Optical Engineering, 50, 1-7. http://dx.doi.org/10.1117/1.3530085

[13] Hamed, A.M. (2011) Computer Generated Quadratic and Higher Order Apertures and Its Application on Numerical 
Speckle Images. Optics and Photonics Journal, 1, 43-51. http://dx.doi.org/10.4236/opj.2011.12007

[14] Hamed, A.M. (2014) Study of Graded Index and Truncated Apertures Using Speckle Images. Precision Instrument and Mechanology (PIM), 3, 144-152.

[15] Born, M. and Wolf, E. (1964) Principles of Optics, Electromagnetic Theory of Propagation, Interference and Diffraction of Light. 2nd Edition, 325. 